\title{
Cognitive rehabilitation for early stage Alzheimer's disease: a pilot study with an Irish population
}

\author{
M. E. Kelly ${ }^{1, *}$, B. A. Lawlor ${ }^{2}$, R. F. Coen ${ }^{3}$, I. H. Robertson ${ }^{2}$ and S. Brennan ${ }^{2}$ \\ ${ }^{1}$ Department of Psychology, John Hume Building, Maynooth University, Co. Kildare, Ireland \\ ${ }^{2}$ Institute of Neuroscience, Trinity College Dublin, Dublin, Ireland \\ ${ }^{3}$ Mercer's Institute for Successful Ageing, Clinical Research Facility, St. James Hospital, Dublin, Ireland
}

\begin{abstract}
Objectives. Research shows that cognitive rehabilitation (CR) has the potential to improve goal performance and enhance well-being for people with early stage Alzheimer's disease (AD). This single subject, multiple baseline design (MBD) research investigated the clinical efficacy of an 8-week individualised CR intervention for individuals with early stage $\mathrm{AD}$.
\end{abstract}

Methods. Three participants with early stage AD were recruited to take part in the study. The intervention consisted of eight sessions of 60-90 minutes of CR. Outcomes included goal performance and satisfaction, quality of life, cognitive and everyday functioning, mood, and memory self-efficacy for participants with AD; and carer burden, general mental health, quality of life, and mood of carers.

Results. Visual analysis of MBD data demonstrated a functional relationship between CR and improvements in participants' goal performance. Subjective ratings of goal performance and satisfaction increased from baseline to post-test for three participants and were maintained at follow-up for two. Baseline to post-test quality of life scores improved for three participants, whereas cognitive function and memory self-efficacy scores improved for two.

Conclusions. Our findings demonstrate that CR can improve goal performance, and is a socially acceptable intervention that can be implemented by practitioners with assistance from carers between sessions. This study represents one of the promising first step towards filling a practice gap in this area. Further research and randomised-controlled trials are required.

Received 10 October 2016; Revised 23 March 2017; Accepted 18 May 2017

Key words: Alzheimer's disease, cognitive rehabilitation, intervention, multiple baseline design.

There is growing evidence that in the early stages of dementia, the brain retains cognitive and neural plasticity (Fernandez-Ballesteros et al. 2005), and can recruit additional neural networks to compensate for damage caused to other areas of the brain (Grady et al. 2003). Supporting research shows that although memory and other cognitive functions are centrally affected by dementia, people with early stage dementia have retained cognitive and functional abilities and are often capable of new learning (Clare \& Wilson, 2004). These findings indicate that cognitive interventions for dementia, aimed at delaying the progression of symptoms or improving functioning, might be most advantageous when implemented earlier in the disease course. Research shows that early cognitive interventions can build on preserved aspects of memory, and develop ways of compensating for impairments; thus increasing the

\footnotetext{
* Address for correspondence: M. E. Kelly, Department of Psychology, Room SF21, 2nd Floor John Hume Building, North Campus, Maynooth University, Co. Kildare, Ireland

(Email: Michelle.E.Kelly@nuim.ie)
}

possibility of enhancing or maintaining functioning and reducing excess disability (Clare, 2008).

Early interventions for people with dementia typically consist of either cognitive training or cognitive rehabilitation (CR). Cognitive training targets underlying impairment and involves guided practise on a standard set of cognitive tasks (Martin et al. 2011). Although some studies show that cognitive training might benefit cognitive performance, results are inconsistent, and benefits have not been shown to transfer to everyday or functional abilities (Sitzer et al. 2006; Bahar-Fuchs et al. 2013). CR may offer a promising alternative to cognitive training because it targets functional disability through individualised, goal-focused interventions, that draw on retained strengths to support adaptive behaviour (Clare et al. 2013). The aim of CR is to enable individuals experiencing progressive cognitive decline to achieve their optimum levels of well-being by improving performance on personally relevant goals. The CR intervention typically focuses on restoration of function, compensatory strategies, and environmental modification (Clare, 2008). 
Restoration of function aims to build on the individual's retained abilities in order to promote new learning or relearning (Clare et al. 2013). An array of instructional strategies can be used to facilitate new learning including spaced retrieval, shaping and chaining (Skinner, 1953), forward and backward cues, mnemonics, semantic elaboration, action-based encoding, and prompting and fading (see Clare, 2008; Cooper et al. 2007 for more detailed descriptions). During intervention sessions, instructional strategies can be applied using an errorless learning (EL) paradigm (Terrace, 1963), with strategies that require effortful or effortless processing (Clare \& Wilson, 2004). EL is used to reduce or eliminate errors during learning trials, and has been shown to be beneficial for some participants with Alzheimer's disease (AD) under certain conditions; particularly when teaching face-name associations, personal information, or using memory aids (e.g. Baddeley \& Wilson, 1994; Clare et al. 2002; Clare \& Wilson, 2004). Effortful processing on the other hand, demands more active encoding of to-belearned information than effortless processing; and may also be a useful technique (Clare \& Wilson, 2004; Dunn \& Clare, 2007). The most recent evidence suggests that increasing the level of effort required at encoding may be more important than focusing on eliminating errors (Dunn \& Clare, 2007); although effortful processing can be applied within an EL paradigm (Clare \& Wilson, 2004).

Literature on precision teaching (PT) has shown that it is a highly effective teaching strategy to promote learning and fluent responding in clinical and educational contexts with younger populations (Lindsley, 1991; Kubina et al. 2000). Despite this, very few studies have considered the utility of this method as a cognitive rehabilitative strategy for people with AD (Johnson-Talbert \& Cooper, 1992). In light of existing evidence, it may be worthwhile to incorporate PT as part of a rehabilitative intervention targeting restoration of function.

Compensatory strategies encourage the use of memory aids to facilitate novel ways of performing cognitive tasks and provide practical solutions for managing cognitive deficits. Environmental modification might include rearranging an individual's environment to promote efficiency, introducing electronic or computer equipment to support independent functioning, or using visual prompts or schedules to build routines (Clare, 2008). During CR, it can also be beneficial to explore the person's ways of managing stress or anxiety, and to provide relevant practice in simple relaxation techniques (Suhr, 1999).

Evidence from single-case and small-group intervention studies show that $C R$ has the potential to improve performance on personally relevant goals or targets that present challenges to everyday living (e.g. remembering appointments); as well as enhancing well-being, and promoting active involvement in daily life for people with mild cognitive impairment (Clare et al. 2009; O'Sullivan et al. 2015) and early stage dementia (Clare et al. 2003; Clare \& Wilson, 2004; Clare, 2010). Two randomised-controlled trials (RCTs) examined the impact of CR in early stage AD and showed that compared with alternative treatments or no treatment controls, CR improved ratings of goal performance and satisfaction, and caregiver's self-reported quality of life (Clare et al. 2010; Kurz et al. 2012). A recent Cochrane review concluded, however, that further studies of $\mathrm{CR}$ are required to provide more definitive evidence (Bahar-Fuchs et al. 2013). There is only one known published study in Ireland of CR with people with mild cognitive impairment (O'Sullivan et al. 2015); and none with people with early stage AD.

The content of CR interventions is determined by individual goals selected by each participant, and as such, goals generally differ across participants (Clare et al. 2003). For this reason, a single-subject design approach is appropriate and beneficial as it allows for objective analysis of individual intervention outcomes (Horner et al. 2005). In addition, information about specific deficits and participants' approaches to goals can be lost in RCTs and group designs, as only information applicable to all participants can be reported (e.g. subjective ratings of goal performance and satisfaction; standardised measures of cognitive function); an issue which is ameliorated by using a single-subject design (Horner et al. 2005; Shadish \& Rindskopf, 2007).

This pilot study aims to develop and evaluate a CR intervention for three older adults with early stage AD. We examined the impact of CR on actual and self-rated goal performance, self-rated goal satisfaction, quality of life, cognitive and everyday functioning, mood, and memory self-efficacy for people with early stage AD; and also on carer burden, general mental health, quality of life, and mood of carers.

\section{Method}

\section{Participants}

Five participants were initially identified, through the Alzheimer Society of Ireland, to take part in the study but two were excluded after screening. Participants were required to have a formal National Institute of Neurological and Communicative Disorders and Stroke and the Alzheimer's Disease and Related Disorders Association diagnosis of $\mathrm{AD}$, be community dwelling with a carer willing to participate, and be able to give informed consent. Participants were screened using the Mini Mental Status Examination (MMSE; Folstein et al. 1975; range, 0-30, lower scores indicate poorer cognitive function), and were required to score between 18 and 24 to be considered for inclusion. Excluded participants received alternative intervention materials and information regarding services 
for people with mild cognitive impairment/dementia. The three participants included in the study consisted of two men and one woman, aged between 60 and 85 years with a mean age of 73.33 years $(\mathrm{SD}=12.58)$; all had received an $\mathrm{AD}$ diagnosis from a neurologist. At screening, participant one (P1) scored 18, participant two (P2) scored 24, and participant three (P3) scored 20 on the MMSE. The mean MMSE score was 20.66 (SD = 3.05).

\section{Ethical approval}

The research methodology and study procedures were granted ethical approval by St. James Hospital Research Ethics Committee.

\section{Design}

The study was conceptualised as a pre-post-test, singlecase multiple baseline design (MBD). Scores on self- and informant-rating scales and on cognitive test measures were obtained at baseline and post-test. A selection of measures were also administered at follow-up. In addition, individual performances on intervention goals were recorded during baseline and intervention sessions, and evaluated using an MBD. The MBD is the most commonly used single-subject design in psychology and education (Shadish \& Sullivan, 2011); and is a useful method of evaluating intervention effects, as it allows for causal inferences to be made about effects of the independent variable on the dependent variable (Cooper et al. 2007). The research was guided by the Single-Case Experimental Design (SCED) (Tate et al. 2008) and The Revised Risk of Bias in N of 1 Trials (RoBiNT) scales (Tate et al. 2013), designed to measure methodological quality in single-subject design studies. The study met eight of the 11 criteria on the SCED scale; and scored 21 out of a possible 30 points on the RoBiNT scale. Assessments and CR sessions were conducted in participants' homes. All other aspects of the research planning and analysis took place at the NeuroEnhancement for Independent Lives (NEIL) Programme in the Institute of Neuroscience, Trinity College Dublin.

\section{Procedure}

The study comprised of five phases: (1) baseline assessments, (2) goal identification, (3) goal baseline measurement, (4) CR intervention sessions, and (5) post-test assessments (post-intervention and 6-week follow-up). Baseline assessments and goal identification were conducted on weeks 1 and 2; the CR intervention began on week 3 and continued for 8 weeks; post-test outcomes were measured at week 11 (1 week after the final intervention session); and follow-up assessments were conducted 6 weeks after the intervention concluded, at week 17 .
Phases 1 and 5: Baseline and post-test assessments

The Repeatable Battery for the Assessment of Neuropsychological Status (RBANS; Randolph et al. 1998; alternate forms $A$ and $B$ used, lower scores indicate poorer cognitive function) was administered to establish a neuropsychological profile of participants' strengths and weaknesses. The use of either Form A or Form B at baseline was randomly selected for each participant, and subsequently the alternate form was used at posttest and follow-up. Remaining assessments included the Montreal Cognitive Assessment (MoCA; Nasreddine et al. 2005; range 0-30, lower scores indicate poorer cognitive function; test-retest reliability, 0.92), Quality of Life in AD (QoL-AD; Logsdon et al. 1999; range 13-52, lower scores indicate lower QoL) self and informant, Instrumental Activities of Daily Living (Lawton \& Brody, 1969, range 0-8, lower scores indicate poorer everyday functioning), Hospital Anxiety and Depression Scale (HADS; Zigmond \& Snaith, 1983; anxiety range 0-21; depression range 0-21, lower scores indicate better mood), and Memory Awareness Rating Scale (MARS; Clare et al. 2002; self- and informant memory functioning subscale; range $0-52$, lower scores indicate poorer perception of memory functioning). Self-rated goal performance and satisfaction were measured using the structured Bangor Goal-Setting Interview (BGSI; Clare et al. 2002; performance range 1-10, 10 = able to carry out extremely well with no difficulty; satisfaction range 1-10, 10 = extremely satisfied with this level of performance); goal performance was also measured using an objective measure of percentage of correct responses.

For carers, assessments included the Zarit Burden Interview (22-item; Zarit et al. 1985; range 0-22, lower scores indicate less burden), HADS, General Health Questionnaire-12 (GHQ-12; Goldberg, 1992; range 0-12, lower scores indicate better general mental health), and World Health Organization Quality of Life Assessment, short version (WHOQOL-BREF; Bonomi et al. 2000; range 26-130, lower scores indicate poorer QoL). All outcomes were measured at baseline and post-intervention; only the RBANS and the QoL-AD were measured at the 6-week follow-up (see Tables 2-4). Test--retest reliability for the RBANS was reported at 0.80 (Dong et al. 2013) and for the QoL-AD was reported at 0.76 for patients and 0.92 for caregivers (Ready \& Ott, 2003).

\section{Phase 2: Goal identification}

Three to four personal rehabilitative goals were identified for each participant through discussions with the participant and their carer, and using the structured BGSI (Clare et al. 2002). Goals were personally relevant for each participant and reflected areas that were either causing difficulty or where the participant would like to see improvement (see Table 1). 
Table 1. Details of the cognitive rehabilitation (CR) intervention strategies implemented for each goal across the three participants

\begin{tabular}{|c|c|c|}
\hline & Goals & Description of intervention \\
\hline \multirow[t]{4}{*}{ P1 } & Memory aids & $\begin{array}{l}\text { A whiteboard with information about the day, date, and appointments was placed on a wall in the participant's primary living area. The participant also wore a watch. } \\
\text { At random intervals during intervention sessions the participant was asked the time or about an upcoming appointment and then immediately prompted to check } \\
\text { the whiteboard or watch. Verbal encouragement was provided with a reminder that memory aids can be used when unsure of information. Prompts were gradually } \\
\text { faded with a progressive time delay of } 1,2,3,5,8,10 \text { seconds. A standard prompt was also provided in response to spontaneous questions. The number of correct } \\
\text { unprompted responses per session was recorded }\end{array}$ \\
\hline & Using the phone & $\begin{array}{l}\text { The target activity was divided into five steps }(1=\text { go to the phone; } 2=\text { find the number in the phonebook; } 3=\text { pick up the phone; } 4=\text { dial the number; } 5=\text { hang up the } \\
\text { phone) and taught using backward chaining, prompting and fading. Verbal and gestural prompts were delivered for steps } 1-5 \text { in the chain. Prompts were } \\
\text { subsequently faded back for step } 5 \text {. When step } 5 \text { was completed by the participant independently, prompts were faded back for step } 4 \text {, and so on until all steps in the } \\
\text { chain could be completed by the participant independently. Action-based encoding was implemented throughout. As mastery increased during intervention } \\
\text { sessions, carer practice sessions incorporated additional prompting and reinforcement for using the phone to attempt to increase spontaneous phone use }\end{array}$ \\
\hline & Face-name recall & $\begin{array}{l}\text { Recall of five names was targeted using verbal elaboration of information about each person, mnemonics, learning the name using forward cues, and rehearsing the } \\
\text { name using spaced retrieval at the time intervals } 0,30 \text { seconds, 1, 2,5,10 minutes. If an incorrect or no response was given at a certain interval, the persons' name was } \\
\text { provided and the interval was repeated. If this occurred again, the interval was halved. Criterion was reached with correct recall after } 10 \text { minutes. Only one new item } \\
\text { was trained per session with probe test trials of all items per session }\end{array}$ \\
\hline & Relaxation & $\begin{array}{l}\text { A relaxation compact disc (CD) was given to the participant and played at regular intervals during intervention sessions. The carer also encouraged the participant to } \\
\text { listen to the } \mathrm{CD} \text { and engage in relaxation strategies (e.g. passive muscle relaxation) between sessions }\end{array}$ \\
\hline \multirow[t]{4}{*}{ P2 } & Number recall & $\begin{array}{l}\text { Recall of four numbers, namely three number plates of family cars and one mobile phone number were targeted by chunking numbers together (i.e. conceptualising } \\
3-5-6 \text { as 356), selecting verbal mnemonics for each number, learning the number using forward cues, and rehearsing the number using spaced retrieval, as specified } \\
\text { for P1's Face-name recall goal }\end{array}$ \\
\hline & Face-name recall & $\begin{array}{l}\text { Recall of } 13 \text { famous face-name associations was targeted using direct instruction and precision teaching. During intervention sessions, all photographs were placed on } \\
\text { the table and names identified. The researcher asked the participant to relay } 2-3 \text { pieces of information about each person and develop mnemonics. The participant } \\
\text { then had to 'point to_-' each person. This was repeated } 2-3 \text { times. Photos were then shuffled and placed on the table one-by-one to be named by the participant. The } \\
\text { correction procedure involved naming the person in the picture and recalling the earlier discussed pieces of information. Criterion was reached when all names were } \\
\text { correctly recalled. At the end of each session a } 1 \text { minute fluency probe was conducted to assess the number of names correctly recalled per minute. The participant } \\
\text { tracked their own performance }\end{array}$ \\
\hline & Verbal fluency & $\begin{array}{l}\text { Verbal fluency was targeted using specific practice on tasks including phonemic and semantic fluency. Intervention sessions also included identifying common nouns, verbs, } \\
\text { adjectives, and prepositions, and attempting to select alternative words with the same or similar meaning. Only self-rated performance and satisfaction were measured for } \\
\text { this goal as it was not possible to measure instances of correct responding }\end{array}$ \\
\hline & Relaxation & A relaxation $\mathrm{CD}$ was given to the participant to use between sessions \\
\hline \multirow[t]{4}{*}{ P3 } & Familiar face-name & Recall of five names was targeted using the learning strategies outlined for P1's face-name recall procedure \\
\hline & Famous face-name & Recall of five famous face-name associations was targeted using the learning strategies outlined for P2's face-name recall procedure \\
\hline & $\begin{array}{l}\text { Using a mobile } \\
\text { phone }\end{array}$ & $\begin{array}{l}\text { Recall of the sequence required to make a phone call was targeted using forward chaining, prompting and action-based encoding. The behaviour was divided into six } \\
\text { steps }(1=\text { decide who to call; } 2=\text { unlock the phone by pressing the middle button (unlock) then *; } 3=\text { press the 'down' arrow on the middle button; } 4=\text { type in the } \\
\text { letters of the name you want to call; } 5=\text { press the green call button to call; } 6=\text { when the call is over, press the red button to hang up). Intervention sessions consisted } \\
\text { of repeated practise naming each step in the chain. Verbal and gestural prompts were provided when required along with a verbal mnemonic assist recall for the red } \\
\text { and green button functions (e.g. 'green to go', 'red to stop'). Test trials recorded correct independent responses when asked to make a call }\end{array}$ \\
\hline & Using a Sat Nav & $\begin{array}{l}\text { An instruction sheet with written and visual cues was created to help the participant to effectively use the Sat Nav for obtaining directions to pre-specified locations. } \\
\text { Intervention sessions took place in the participant's car using the instructions sheet. The participant practised using the Sat Nav outside of CR sessions, while driving } \\
\text { to various locations. Only self-rated performance and satisfaction were measured for this goal as it was not possible to measure instances of correct responding }\end{array}$ \\
\hline
\end{tabular}
subsequently faded back for step 5 . When step 5 was completed by the participant independently prompts were faded back for step 4 , and so on until all steps in the

Recall of five names was targeted using verbal elaboration of information about each person, mnemonics, learning the name using forward cues, and rehearsing the was trained per session with probe test trials of all items per session

listen to the $C D$ and engage in relaxation strategies (e $g$ passive muscle relaxation) between sessions (1) 年

rbal fluency was targeted using specific practice on tasks including phonemic and semantic fluency. Intervention sessions also included identifying common nouns, verbs, 作 this goal as it was not possible to measure instances of correct responding

Famous face-name Recall of five famous face-name associations was targeted using the learning strategies outlined for P2's face-name recall procedure

Using a mobile Recall of the sequence required to make a phone call was targeted using forward chaining, prompting and action-based encoding. The behaviour was divided into six steps $(1=$ decide who to call; $2=$ unlock the phone by pressing the middle button (unlock) then *; $3=$ press the 'down' arrow on the middle button; $4=$ type in the letters of the name you want to call; $5=$ press the green call button to call; $6=$ when the call is over, press the red button to hang up). Intervention sessions consisted instruction sheet with written and viug che participant to effectively use the Sat Nav for obtaining directions to pre-specified locations. to various locations. Only self-rated performance and satisfaction were measured for this goal as it was not possible to measure instances of correct responding 


\section{Phase 3: Goal baseline measurement}

Where possible on individual goals, baseline, and intervention data were recorded as percentage of correct responses, and plotted MBD graphs (see Figs. 1-3). Simultaneous baseline data were gathered on two or more goals per participant. After a stable baseline was observed for one goal, the CR intervention was applied while baseline conditions were maintained for the remaining goals. After change in responding was observed for the first goal, the CR intervention was applied sequentially to the next goal with a stable baseline (Cooper et al. 2007). The data were recorded during clinical sessions by the first author.

\section{Phase 4: Intervention}

CR intervention methods, informed by $C R$ and behavioural intervention research literature, were devised to address participants' identified goals. The first author implemented the CR interventions with all three participants as she was a Board Certified Behaviour Analystdoctoral level, and had extensive experience with the implementation of evidence-based rehabilitative interventions. Intervention sessions were conducted for 60-90 minutes once per week over 8 weeks. Participants were encouraged to work on goals between sessions. Intervention sessions took place in participant's homes to facilitate learning in the everyday setting. Where possible, a carer or family member joined in the last 10 minutes of each session. This involved reviewing the content of the session, agreeing homework, and discussing how to facilitate progress with personal goals. Performance data were recorded as percent correct at each session (as above), and typically each session included 10 intervention trials. The subsequent data were graphed on MBD graphs (Figs. 1-3).

Overall, the CR intervention incorporated techniques for learning new information, encouraged the use of learning strategies and memory aids every day, and encouraged relaxation. Memory rehabilitative strategies included the use of verbal and visual mnemonics, forward cueing, spaced retrieval, direct instruction, and PT. Strategies for improving procedural memory included action-based encoding, chaining, prompting, and fading (see Table 1). All interventions incorporated an EL paradigm by encouraging participants not to guess, but rather to respond with 'I don't know' or not respond if unsure of an answer. Test sessions were conducted at the end of each intervention session, and data were recorded on MBD graphs. Where possible, carers attended the end of each session and were provided with a summary detailing areas covered, techniques used, and agreed practice. At the conclusion of the 8-week intervention each participant received a final report summarising goal performance, and effective and preferred strategies.

\section{Results}

\section{Visual analysis of goal performance}

Data were recorded during each session on participants' performance on selected goals (proportion of correct responses per session). The data were then graphed on MBD graphs (see Figs. 1-3). Within and between condition analyses examined trend, level, and stability of data in order to evaluate intervention effects (Lane \& Gast, 2014). Stability was assessed using a stability envelope, consisting of two parallel lines drawn above and below the median line; the distance between the two lines shows how much variability is allowed for the data to be considered stable (see Gast, 2010; Lane \& Gast, 2014 for a more thorough description). Criterion for the stability envelope was set at $70 \%$ due to low numbers of data points across phases. It was not possible to create a stability envelope for phases with all data points at zero. Percentage of nonoverlapping data (PND) was used to quantify the extent to which scores were shared across phases; $100 \%$ non-overlap occurs when post-test values are greater or less than those recorded at baseline with no shared value (Manolov et al. 2016). Although relaxation was targeted as part of the intervention for two participants, it was not appropriate to measure performance across sessions. Similarly, proportion of correct responses could not be measured for the 'fluency' goal for P2, and 'using a Sat Nav' for P3. These goals were instead targeted for general improvement outside of CR sessions.

\section{P1 goal performance}

Evaluation of level change within conditions for each of P1s' three goals indicated that performance was stable during baseline and intervention. Accuracy criterion of $100 \%$ was reached during the intervention for 'memory aids' and 'using the phone'. For the 'face-name recall' goal, performance was variable during baseline and intervention, but also improved to $100 \%$ accuracy in the intervention phase. Split-middle method of trend estimation indicated a contra-therapeutic trend during baseline and an increasing trend in a therapeutic direction during intervention for each of the three goals. The data for 'memory aids' and 'using the phone' were considered stable following application of a stability envelope to trend lines as $89 \%$ of data points in the intervention phase fell inside the trend stability envelope. Baseline data for the 'face-name recall' goal were variable as $57.14 \%$ of baseline data points and but intervention data were stable as $71.4 \%$ fell inside the trend stability envelope.

Evaluation of behaviour change between conditions indicated that performance in all three goals changed from a level trend in baseline to an accelerating improving 


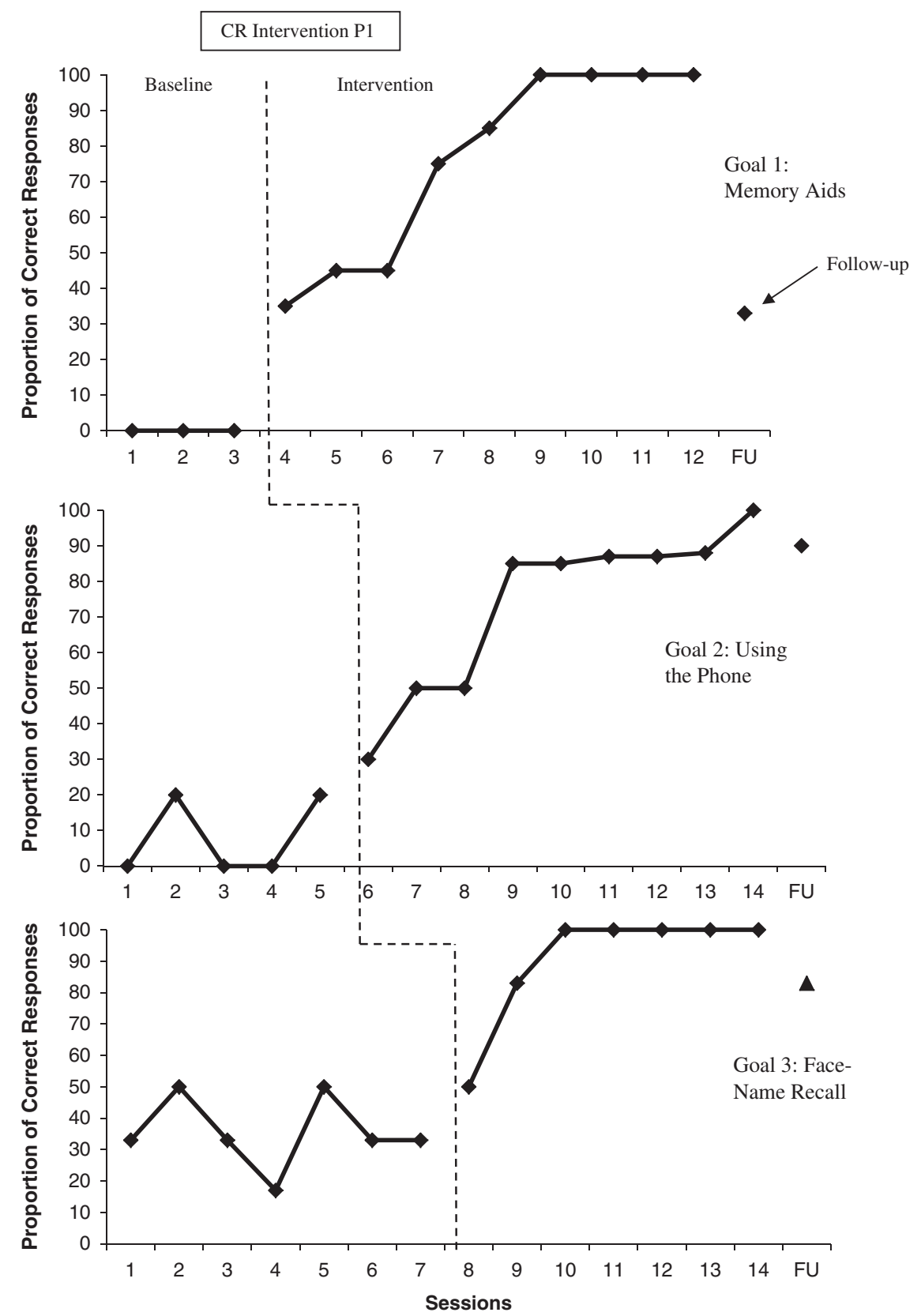

Fig. 1. Participant 1 - memory aids: proportion of correct responses per session, when asked date/time or appointment details the participant checked her watch, calendar, or whiteboard; phone call: proportion of correct responses per session, action of making a call divided into four steps; face-name recall: proportion of correct responses across five names. CR, cognitive rehabilitation.

trend during the intervention. Relative, absolute, median, and mean level change calculations indicated a positive and improving change across conditions; $+45,+35,+85$, +76.11 , respectively, for 'memory aids'; $+40,+10,+85$, +65.55 for 'using the phone'; and $+50,+17,+67,+54.85$ for 'face-name recall'. Calculations of PND indicated that there was $100 \%$ non-overlap of data observed between phases across each of the three goals. Maintenance data show that on the 'memory aids' goal, accuracy dropped from $100 \%$ at the end of the intervention to $33 \%$ at follow-up. Despite this, the participant commented at follow-up 'I use my watch all the time now, but I didn't before'. For 'using the phone', accuracy only dropped $10-90 \%$ at follow-up. For 'face-name recall', accuracy dropped from $100 \%$ at intervention to $83 \%$ at follow-up.

\section{P2 goal performance}

Evaluation of level change within conditions for both of P2's goals indicated that performance was stable 


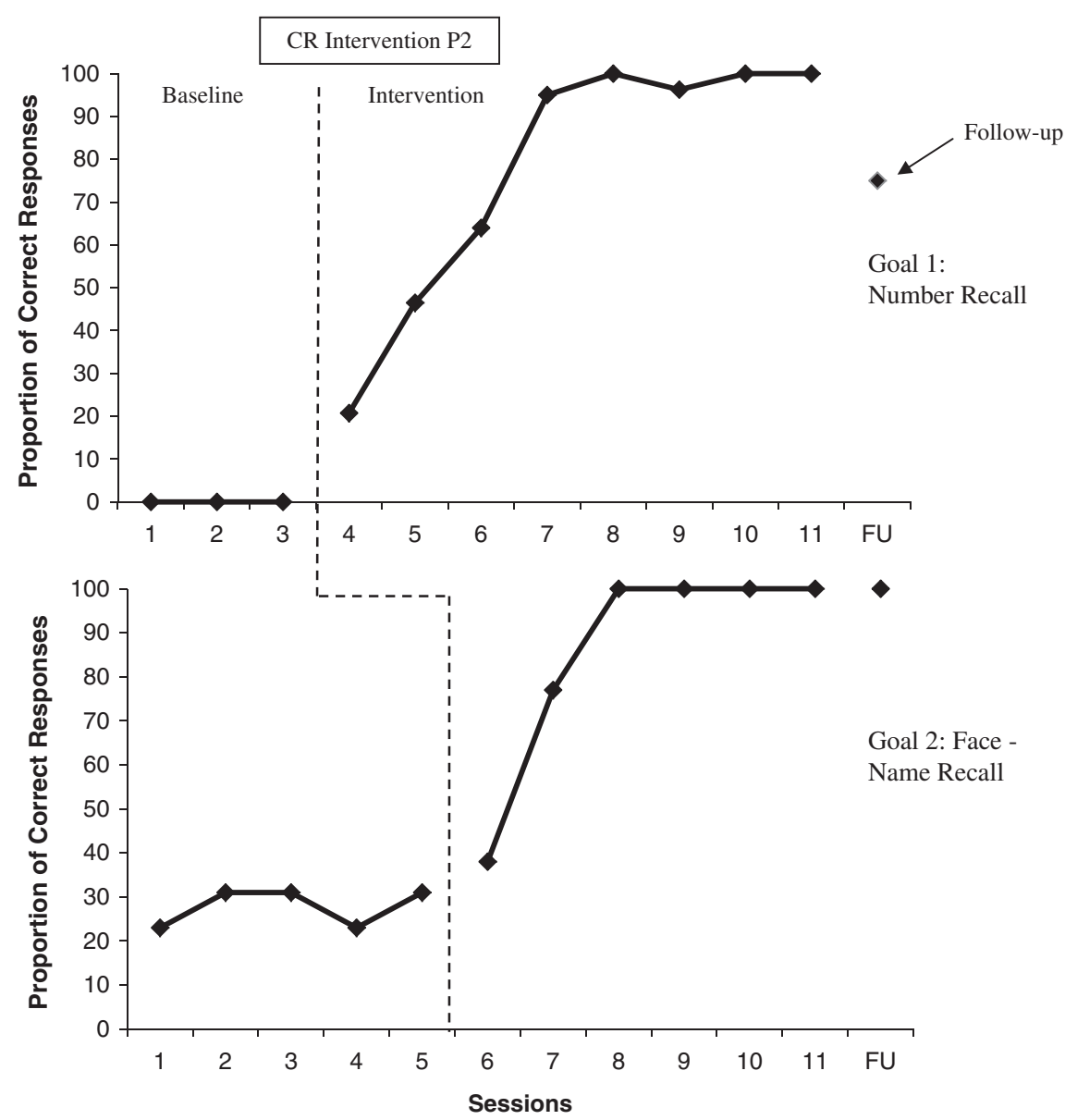

Fig. 2. Participant 2 - number recall: proportion of correct responses per session, recall of four numbers; face-name recall: proportion of correct responses per session, recall of 13 famous face-name associations. CR, cognitive rehabilitation.

during baseline and intervention for 'number recall' and 'face-name recall' but was variable for the intervention phase of 'face-name recall'. Performance on both goals reached $100 \%$ accuracy. Split-middle method of trend estimation indicated a contratherapeutic trend during baseline and an increasing trend in a therapeutic direction during intervention for both goals. When the trend stability envelope was applied, 75\% of intervention data for 'number recall', and $100 \%$ of baseline data and $67 \%$ of intervention data points for 'face-name recall' fell within the envelope.

Evaluation of behaviour change between conditions indicated that performance in both goals changed from a level trend in baseline to an accelerating improving trend during the intervention. Relative, absolute, median, and mean level change calculations indicated a positive and improving change across conditions; $+55.25,+20.75,+95.62,+77.81$, respectively, for 'number recall' and $+50,+7,+69,+58.03$, respectively, for 'face-name recall'. Calculations of PND indicated that there was $100 \%$ non-overlap of data observed between phases. Maintenance data show that on the 'number recall' goal, accuracy dropped from $100 \%$ at the end of the intervention to $75 \%$ at follow-up. For 'face-name recall', intervention effects were maintained at $100 \%$ accuracy at the 6-week follow-up.

For the 'fluency' goal, P2 reported improvements in conversational fluency; specifically 'I have noticed an improvement... this has given me the confidence to take my time and try to think of the words I want to use, instead of just not saying them'. Similarly for the relaxation goal, P2 reported listening to the relaxation $\mathrm{CD}$ during times of stress, and finding it beneficial to 'escape and relax'.

\section{P3 goal performance}

Evaluation of level change within conditions for P3's three goals indicated that performance was stable during baseline and intervention for 'familiar face-name recall' but was variable for both phases of the 'famous face-name recall' and 'using a mobile phone' goals. Performance on all goals reached $100 \%$ accuracy. Split-middle method of trend estimation indicated a contra-therapeutic trend during baseline and an 


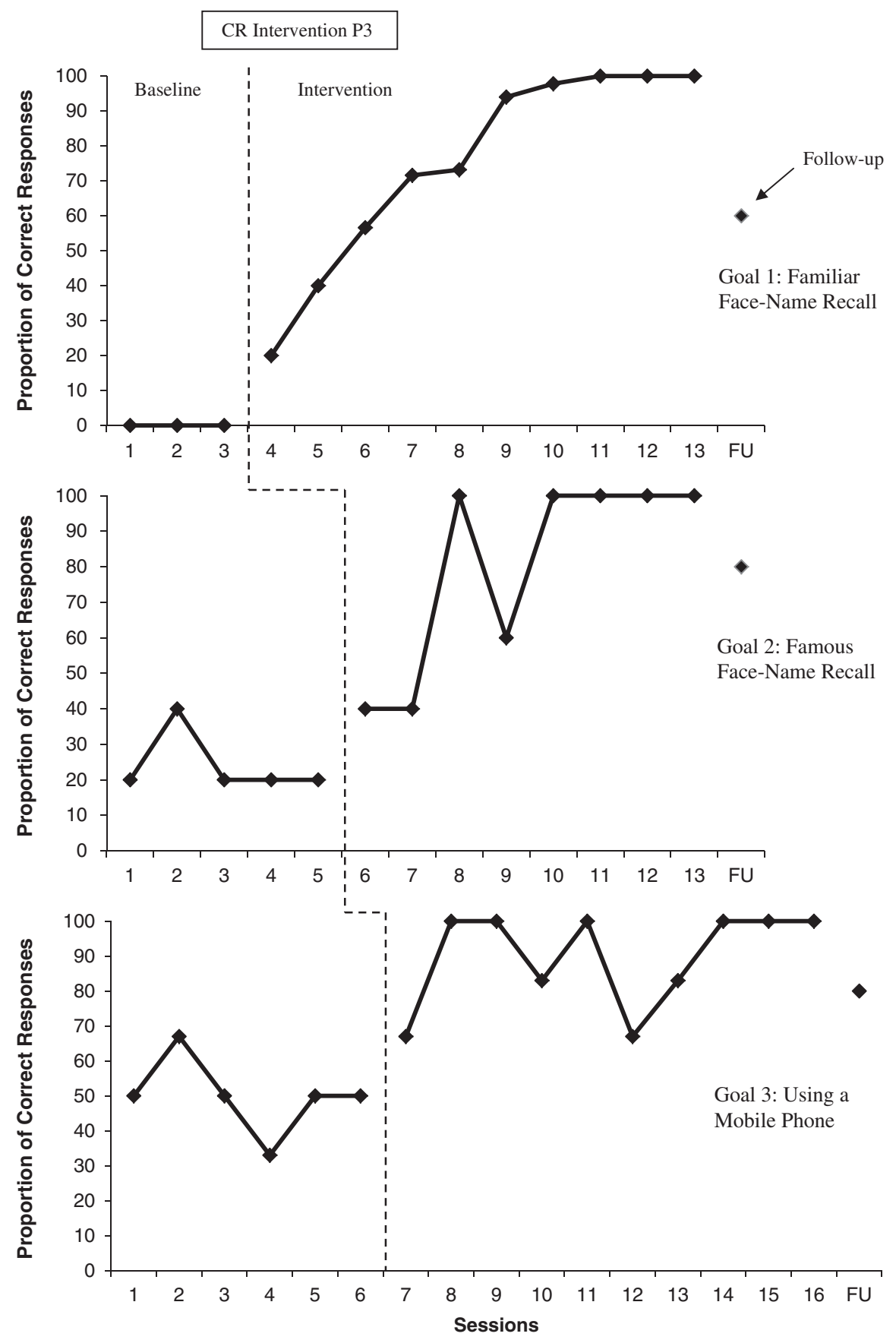

Fig. 3. Participant 3 - familiar face-name recall: proportion of correct responses, recall of five familiar face-name associations; famous face-name recall: proportion of correct responses per session, recall of five famous face-name associations; using a mobile phone: proportion of correct responses per session, action of using the phone divided into six steps. CR, cognitive rehabilitation.

increasing therapeutic trend during the intervention for all goals. When the trend stability envelope was applied, $70 \%$ of intervention data for 'familiar face-name recall'; $80 \%$ of baseline data and $62.5 \%$ of intervention data points for 'famous face-name recall'; and $67 \%$ of baseline and $60 \%$ of intervention data for 'using a mobile phone' fell within the envelope.
Evaluation of behaviour change between conditions indicated that performance in all goals changed from no trend in baseline to an accelerating improving trend during the intervention. Relative, absolute, median, and mean level change calculations indicated a positive and improving change across conditions; $+56.6,+20$, +83.6 , +75.32, respectively, for 'familiar face-name 
Table 2. Performance and satisfaction scores (minimum score $=1$; maximum score $=10$ ), including means and SD across goals for each participant at baseline, post-test and follow-up

\begin{tabular}{|c|c|c|c|c|c|c|c|c|c|}
\hline \multirow[b]{2}{*}{ Goals } & \multicolumn{3}{|c|}{ Self-performance } & \multicolumn{3}{|c|}{ Self-satisfaction } & \multicolumn{3}{|c|}{ Informant performance } \\
\hline & BL & PT & FU & BL & PT & FU & $\mathrm{BL}$ & PT & FU \\
\hline \multicolumn{10}{|l|}{ P2 } \\
\hline Phone calls & 1 & 2 & 8 & 1 & 2 & 8 & 1 & 2 & 3 \\
\hline FN recall & 1 & 3 & 5 & 1 & 2 & 5 & 1 & 2 & 5 \\
\hline Memory aids & 8 & 8 & 10 & 8 & 8 & 8 & 8 & 8 & 7 \\
\hline Relaxation & 2 & 7 & 7 & 2 & 7 & 9 & 1 & 3 & 9 \\
\hline Total & 12 & 20 & 30 & 12 & 19 & 30 & 11 & 15 & 24 \\
\hline Mean (SD) & $3(3.37)$ & $5(2.94)$ & $7.5(2.08)$ & $3(3.37)$ & $4.75(3.20)$ & $7.5(1.73)$ & $2.75(3.5)$ & $3.75(2.87)$ & $6(2.58)$ \\
\hline \multicolumn{10}{|l|}{ P2 } \\
\hline Number recall & 3 & 9 & 10 & 3 & 10 & 10 & 2 & 9 & 5 \\
\hline FN recall & 3 & 10 & 9 & 2 & 10 & 10 & 4 & 8 & 4 \\
\hline Fluency & 4 & 8 & 8 & 4 & 10 & 9 & 3 & 5 & 3 \\
\hline Relaxation & 4 & 8 & 8 & 4 & 8 & 10 & 1 & 8 & 7 \\
\hline Total & 14 & 35 & 35 & 13 & 38 & 39 & 10 & 30 & 19 \\
\hline Mean (SD) & $3.5(0.58)$ & $8.75(0.96)$ & $8.75(0.96)$ & $3.25(0.96)$ & $9.5(1)$ & $9.75(0.5)$ & $2.5(1.29)$ & $7.5(1.73)$ & 4.75 (1.71) \\
\hline \multicolumn{10}{|l|}{ P3 } \\
\hline Use Sat Nav & 5 & 6 & 6 & 4 & 6 & 6 & 4 & 5 & 5 \\
\hline Mobile phone & 7 & 8 & 8 & 6 & 7 & 7 & 5 & 6 & 7 \\
\hline Fs FN recall & 8 & 10 & 7 & 6 & 8 & 5 & 6 & 9 & 8 \\
\hline Fr FN recall & 6 & 9 & 4 & 5 & 8 & 4 & 6 & 9 & 8 \\
\hline Total & 26 & 33 & 25 & 21 & 29 & 22 & 21 & 29 & 28 \\
\hline Mean (SD) & $6.5(1.29)$ & $8.25(1.71)$ & $6.25(1.71)$ & $5.25(0.96)$ & $7.25(0.96)$ & 5.5 (1.29) & $5.25(0.96)$ & $7.25(2.06)$ & $7(1.41)$ \\
\hline
\end{tabular}

BL, baseline; PT, post-test; FU, follow-up; FN, face-name recall; Fs FN, famous face-name recall; Fr FN, familiar face-name recall

Table 3. Mean difference, including standard deviation (SD) and 95\% confidence intervals (CI), between total ratings on $12 \mathrm{CR}$ goals (3 participants $\times 4$ goals) at baseline (BL), post-test (PT), and follow-up (FU) for self-performance, self-satisfaction, and informant performance.

\begin{tabular}{|c|c|c|c|}
\hline Goal ratings & Mean difference & $\mathrm{SD}$ & $95 \%$ CI \\
\hline $\begin{array}{l}\text { Self-performance } \\
\text { BL-PT }\end{array}$ & -3.00 & 2.21 & $-4.41,-1.59$ \\
\hline $\begin{array}{l}\text { Self-performance } \\
\text { BL-FU }\end{array}$ & -3.17 & 2.97 & $-5.06,-1.27$ \\
\hline $\begin{array}{l}\text { Self-satisfaction } \\
\text { BL-PT }\end{array}$ & -3.33 & 2.64 & $-5.01,-1.66$ \\
\hline $\begin{array}{l}\text { Self-satisfaction } \\
\text { BL-FU }\end{array}$ & -3.75 & 3.39 & $-5.90,-1.60$ \\
\hline $\begin{array}{l}\text { Informant Performance } \\
\text { BL-PT }\end{array}$ & -2.67 & 2.31 & $-4.13,-1.20$ \\
\hline $\begin{array}{l}\text { Informant Performance } \\
\text { BL-FU }\end{array}$ & -2.42 & 2.57 & $-4.05,-0.78$ \\
\hline
\end{tabular}

recall'; +50, $+20,+80,+56$, respectively, for 'famous face-name recall'; and $+50,+17,+50,+40$, respectively, for 'using a mobile phone'. Calculations of PND indicated that there was $100 \%$ non-overlap of data observed between phases for goals 1 and 2; and 80\% non-overlap for goal 3 . Maintenance data show that 'familiar face-name recall' accuracy dropped from $100 \%$ at the end of the intervention to $60 \%$ at follow-up; and 'famous face-name recall' and 'using a mobile phone' accuracy dropped to $80 \%$ at follow-up. $\mathrm{P} 3$ reported that the instructions provided for the 'using a Sat Nav' goal were 'useful' although it was unclear how often they were effectively used.

\section{Self-Rated Goal Performance and Satisfaction}

Table 2 illustrates scores including means and standard deviations for self- and informant ratings of goal performance and satisfaction. Baseline to post-test scores for self- and informant ratings increased for all participants. Post-test to follow-up self-rating scores increased for P1, remained the same for $\mathrm{P} 2$, and worsened for P3; whereas informant rating scores increased for P1, but decreased for P2 and P3. Difference scores from baseline to post-test and post-test to follow-up for all goals can be seen in Table 3 .

\section{Cognitive function}

For P1 (baseline MMSE of 18), RBANS total scale score changed from 61 at baseline to 53 at post-test and 54 at follow-up; scores remained less than 1st percentile and were categorised as 'extremely low' (see Table 4). For P2 (baseline MMSE of 24), RBANS total scale score 
Table 4. Cognitive test scores at baseline and follow-up; alternate versions of the Repeatable Battery for the Assessment of Neuropsychological Status (RBANS) were used

\begin{tabular}{|c|c|c|c|c|}
\hline Participants & Test & Baseline & Post-test & Follow-up \\
\hline \multirow[t]{3}{*}{ P1 } & MMSE & 18 & & \\
\hline & $\mathrm{MoCA}$ & 11 & 11 & \\
\hline & RBANS (A-B-A) total scale & 61 & 53 & 54 \\
\hline \multirow[t]{5}{*}{ RBANS } & Immediate memory & 90 & 69 & 81 \\
\hline & Visuospatial/constructional & 84 & 60 & 62 \\
\hline & Language & 40 & 44 & 40 \\
\hline & Attention & 91 & 94 & 94 \\
\hline & Delayed memory & 40 & 40 & 40 \\
\hline \multirow[t]{3}{*}{ P2 } & MMSE & 24 & & \\
\hline & $\mathrm{MoCA}$ & 20 & 23 & \\
\hline & RBANS (A-B-A) total scale & 75 & 82 & 81 \\
\hline \multirow[t]{5}{*}{ RBANS } & Immediate memory & 69 & 81 & 85 \\
\hline & Visuospatial/constructional & 87 & 87 & 81 \\
\hline & Language & 85 & 92 & 83 \\
\hline & Attention & 68 & 79 & 79 \\
\hline & Delayed memory & 90 & 95 & 101 \\
\hline \multirow[t]{3}{*}{ P3 } & MMSE & 20 & & \\
\hline & $\mathrm{MoCA}$ & 17 & 18 & \\
\hline & RBANS (B-A-B) total scale & 64 & 70 & 70 \\
\hline \multirow[t]{5}{*}{ RBANS } & Immediate memory & 49 & 49 & 49 \\
\hline & Visuospatial/constructional & 112 & 126 & 112 \\
\hline & Language & 78 & 78 & 78 \\
\hline & Attention & 79 & 91 & 94 \\
\hline & Delayed memory & 40 & 40 & 48 \\
\hline
\end{tabular}

MMSE, Mini Mental Status Examination; MoCA, Montreal Cognitive Assessment. RBANS total scale scores and index scores for separate cognitive abilities included. Qualitative description of RBANS index scores: 69 and below $=$ extremely low; $70-79=$ borderline; $80-89=$ low average; $90-109=$ average; $110-119=$ high average; $120-129=$ superior.

increased from 75 at baseline (5th percentile) to 82 and 81 at post-test and follow-up (12th percentile), increasing from 'borderline' up to 'low average'. For P3 (baseline MMSE of 20), RBANS total scale scores increased from 64 at baseline (1st percentile) to 70 (2nd percentile) at post-test and follow-up, increasing from 'extremely low' to 'borderline'. Baseline to post-test scores on the MoCA remained at 11 for P1, increased from 20 to 23 for $\mathrm{P} 2$, and increased from 17 to 18 for P3.

\section{Subjective questionnaires}

\section{Participants}

All participants' self-reported quality of life scores improved from baseline to post-test with 6-week followup scores largely maintained (Table 5). Everyday functioning scores (Table 5) showed high levels of functioning for all three participants at baseline, which remained relatively unchanged at post-test. None of the participants reported any significant levels of depression or anxiety at baseline or post-test. Memory self-efficacy scores improved for P1 and P3; and remained unchanged for P2.
Carers

Carer 3 (C3) reported mild anxiety (8-10) at baseline whereas the remaining two carers $(\mathrm{C} 1, \mathrm{C} 2)$ reported no significant levels of either depression or anxiety (Table 5). None of the carers reported any significant levels of anxiety or depression at post-test. All three scored within the normal range (11-12) for general mental health (GHQ-12) at both baseline and post-test. Self-reported quality of life was slightly lower for C1 and $\mathrm{C} 3$ at follow-up compared with baseline but improved for $\mathrm{C} 2$ from baseline to post-test. $\mathrm{C} 1$ and $\mathrm{C} 2$ reported little or no burden (0-20) at baseline which increased to mild to moderate burden (20-40) at posttest, whereas C3 reported mild-moderate burden at both baseline and post-test.

\section{Discussion}

\section{Summary of results}

Overall the $\mathrm{CR}$ intervention was effective in improving subjective and objective measures of goal performance, 
Table 5. Primary outcome score ${ }^{a}$ for quality of life (QoL); secondary outcome scores ${ }^{a}$ for participants including everyday functioning (IADL: Instrumental activities of daily living), mood (HADS: Hospital anxiety and depression scale), memory self-efficacy (MARS-F: Memory assessment rating scale-function subscale) self and informant versions; secondary outcome scores for carers including mood (HADS: Hospital anxiety and depression scale), general mental health (GHQ-12: General health questionnaire- 12 item), quality of life (WHOQOL-BREF: World Health Organization's quality of life questionnaire, brief), and carer burden (Zarit Burden Interview)

\begin{tabular}{|c|c|c|c|c|}
\hline Participant & Test & $\begin{array}{l}\text { Baseline } \\
\text { score }\end{array}$ & $\begin{array}{l}\text { Post-test } \\
\text { score }\end{array}$ & $\begin{array}{l}\text { Follow- } \\
\text { up }\end{array}$ \\
\hline \multirow[t]{7}{*}{ P1 } & QoL-AD (S) & 43 & 45 & 40 \\
\hline & QoL-AD (I) & 35 & 29 & 35 \\
\hline & IADL (I) & 6 & 4 & - \\
\hline & HADS-anxiety & 1 & 4 & - \\
\hline & HADS-depression & 7 & 1 & - \\
\hline & MARS-F (S) & 11 & 23 & - \\
\hline & MARS-F (I) & 22 & 18 & - \\
\hline \multirow[t]{5}{*}{$\mathrm{C} 1$} & HADS-anxiety & 2 & 0 & - \\
\hline & HADS-depression & 0 & 0 & - \\
\hline & GHQ-12 & 6 & 6 & - \\
\hline & WHOQOL-BREF & 121 & 118 & - \\
\hline & Zarit Burden & 14 & 21 & - \\
\hline \multirow[t]{7}{*}{ P2 } & QoL-AD (S) & 47 & 49 & 50 \\
\hline & QoL-AD (I) & 34 & 26 & 33 \\
\hline & IADL (I) & 5 & 6 & - \\
\hline & HADS-Anxiety & 3 & 1 & - \\
\hline & HADS-Depression & 1 & 1 & - \\
\hline & MARS-F (S) & 45 & 45 & - \\
\hline & MARS-F (I) & 31 & 30 & - \\
\hline \multirow[t]{5}{*}{$\mathrm{C} 2$} & HADS-anxiety & 2 & 3 & - \\
\hline & HADS-depression & 0 & 2 & - \\
\hline & GHQ-12 & 7 & 2 & - \\
\hline & WHOQOL-BREF & 108 & 114 & - \\
\hline & Zarit Burden & 20 & 28 & - \\
\hline \multirow[t]{7}{*}{ P3 } & QoL-AD (S) & 42 & 47 & 46 \\
\hline & QoL-AD (I) & 45 & 41 & 44 \\
\hline & IADL (I) & 6 & 6 & - \\
\hline & HADS-anxiety & 0 & 1 & - \\
\hline & HADS-depression & 1 & 0 & - \\
\hline & MARS-F (S) & 38 & 44 & - \\
\hline & MARS-F (I) & 30 & 26 & - \\
\hline \multirow[t]{5}{*}{ C3 } & HADS-anxiety & 8 & 7 & - \\
\hline & HADS-depression & 2 & 1 & - \\
\hline & GHQ-12 & 10 & 11 & - \\
\hline & WHOQOL-BREF & 114 & 110 & - \\
\hline & Zarit Burden & 30 & 29 & - \\
\hline
\end{tabular}

${ }^{\mathrm{a}}$ Higher score indicates poorer results for HADS (poorer mood), GHQ-12 (poorer general mental health), Zarit burden (more burden). Higher score indicates better results for QoL-AD, IADL, MARS-F, WHOQOL-BREF.

subjective ratings of goal satisfaction, and quality of life for all three participants with AD. Visual analysis of goal performance showed a replication of intervention effects across goals for all three participants.
This replication demonstrated a functional relationship between the CR intervention and improved goal performance. Baseline to post-intervention cognitive function and memory self-efficacy scores increased for two participants. There were no improvements in ratings of everyday functioning. There was a slight improvement in anxiety ratings for one carer; but no improvements for carers on any of the remaining measures.

\section{Results in the context of Prior CR Research}

\section{Goal performance}

The results of our study are consistent with those of Clare et al. (2010) who reported RCT data showing that $\mathrm{CR}$ was successful in producing significant improvements in ratings of goal performance and satisfaction; and improving ratings of memory self-efficacy for people with early stage AD. The differences between self- and carer ratings of goal performance may have reflected greater sensitivity to social demand for participants with AD; although it has been argued that all explicit measures, whether self- or informant ratings, are susceptible to socially desirable responding (see Barnes-Holmes et al. 2010 for a discussion). Divergence between self- versus informant evaluations of cognitive performance is common in dementia research (Clare et al. 2002) and is more likely due to participants' awareness of their cognitive ability (Clare et al. 2004). In terms of MBD goal performance data, the intra-subject replication of intervention effects demonstrated a functional relationship between the intervention and goal performance. This provides support for a prior multiple single-case experimental design in which CR yielded significant improvements in the proportion of correct responses on a range of memory-related targets for five out of six participants (Clare et al. 2000). Maintenance of intervention gains as seen in this study are commonly noted (e.g. Clare et al. 2000).

\section{Quality of life and carer burden}

Our findings lend support to the results of Kurz et al. (2012) who reported improvements favouring CR on self-reported quality of life of people with AD. In the current study, however, informant ratings of QoL-AD worsened at follow-up; Clare et al. (2010) showed similar trends on QoL-AD scores. Similarly, in both the current study, and in those of Clare et al. (2010) and Kurz et al. (2012), carers' ratings of burden/stress increased after the $\mathrm{CR}$ intervention. One possible explanation might be that carers were required to take part in intervention sessions, and practise CR strategies with participants between sessions. This may have led to an increased perception of burden. The increased engagement may also have led carers to take greater 
note of the participant's deficits. It may also have been the case that carers felt positively about embarking on the intervention at the outset, and negatively about the removal of social support at the conclusion of the intervention. Future studies could examine whether some form of peer support system between carers, which could be continued after the intervention concluded, may improve carer outcomes.

\section{Generalisation}

Based on the results of their RCT, Clare et al. (2010) suggested that the effects of the CR intervention generalised to goals outside of specific intervention targets. Clare et al. (2000) reported similar findings; one couple in their study began discussing, devising, and apply memory strategies when new situations arose. At the follow-up session in our study, P2 explained how strategies learned in the CR sessions were being applied to different situations daily. Practice between sessions appears to be a contributing factor in the generalisation of the intervention effects to goals not targeted within sessions (Clare et al. 2000, 2010). P2 consistently practised the CR strategies between sessions and would often present the researcher with 'homework' completed. For P1, the intervention for using the phone did not generalise to spontaneous use outside of intervention sessions, although P1's carer did report difficulty with practice. Our findings suggest that the potential for generalisation is maximised for those with higher baseline cognitive performance, who engage in regular practice of the strategies learned.

\section{Activities of daily living (ADLs)}

Consistent with the results of Kurz et al. (2012), we failed to find generalisation of intervention effects to ADLs. Kurz et al. suggested that a reason for this might be low sensitivity of the assessment instruments or lack of appropriate measurement. Another explanation might be that participants were already functioning at very high levels at baseline. This is likely to be the case for many participants with early stage $A D$, therefore further improvement on standardised measures of ADLs may be difficult to achieve. Further studies should include direct assessment of ADLs and investigate whether high-ADL scores are typically maintained after longer-term follow-up periods.

\section{Teaching strategies}

An EL was used with effortful processing for all participants (Clare \& Wilson, 2004). P3 reported liking this strategy and that making mistakes negatively impacted his confidence. Our results also support findings that effortful processing may be useful for CR (Clare \&
Wilson, 2004; Dunn \& Clare, 2007); mnemonics and forward cues for teaching recall goals resulted in lasting improvements. For P1, we used an effortless processing strategy (backward cues) due to anxiety and difficulty with the task. Although performance improved, effects did not generalise. This is consistent with reports that strategies relying on effortless processing may not produce learning gains as effectively as effortful strategies (Clare \& Wilson, 2004). Spaced retrieval was very effective for consolidating to-be-remembered information; this strategy has been consistently recommended for memory rehabilitation (Clare \& Wilson, 2004; Oren et al. 2014). To explore the utility of PT in CR, we incorporated PT for recall goals with P2 and P3. Improvements on goals targeted with PT were comparable with improvements with forward cues, although responses for P3 were more variable with PT versus forward cueing. This preliminary data nevertheless suggests that further exploration of PT for CR is warranted. Participants' abilities, preferences, and goals should be considered before deciding on the most appropriate teaching strategies (Clare \& Jones, 2008).

\section{Suitability of CR for early stage AD}

The CR intervention appears to be most beneficial to those with mild impairments in cognitive function. Similar to earlier CR studies (Clare et al. 2000, 2010, 2013), we selected a minimum score of 18 on the MMSE as criterion for inclusion. Despite the fact that all participants improved on goal performance, only the two participants with MMSE scores $>18$ showed improvements on cognitive test scores. P1's cognitive performance declined at post-test, whereas P2 showed increases on MoCA and RBANS scores, which were maintained at follow-up. Earlier studies with participants with baseline MMSE scores of 24 or above similarly reported improvements in cognitive test scores after the intervention (e.g. Clare et al. 2003, 2009). This suggests that $\mathrm{CR}$ is most beneficial to those in the earlier stages of decline. Our findings support the idea that people with early stage dementia have retained cognitive abilities and are capable of new learning (Clare and Wilson, 2004); and underscore the importance of implementing rehabilitative interventions earlier in the disease course (Clare, 2008; O'Sullivan et al. 2015).

\section{Limitations}

The use of a single-subject design with individualised interventions limits the generalisability of our results; although our pilot data suggest that a CR intervention might be of benefit to some people with early stage AD in Ireland. An individual approach is also clinically relevant, particularly when dealing with such a diverse 
clinical population (Clare et al. 2000). A lack of comparative control group does, however, limit the extent to which one can understand the impact of possible confounds such as attention and general social demand. Although statistical analyses were not appropriate, the visual analysis of the experimental MBD data demonstrated a functional relationship and indicated that the changes in goal performances were likely attributable to the intervention. Future singlesubject research should aim to collect MBD data amenable to statistical analysis (see Shadish, 2014).

It was not possible to record inter-observer agreement (IOA) or procedural integrity data due to limited resources, nor was the therapist blind to the treatment condition of the study. In particular, the lack of IOA for the multiple baseline data was a significant limitation, and should be addressed in future research. Although problematic, data were collected using objective recording where possible, the researcher was qualified to deliver interventions, and training was provided by an experienced clinical neuropsychologist in administering and scoring cognitive assessments before study commencement. Although the issue of limited resources in applied practice may often hamper the design of single-case research, future studies should try where possible, to fully adhere to guidelines as outlined by Kratochwill et al. (2010, 2013), Tate et al. (2014) for improving the overall quality of single-subject research.

\section{Conclusions and recommendations}

Overall, our results provide support for $C R$ as an effective intervention to improve goal performance, satisfaction, quality of life, and cognitive function for people with early stage $\mathrm{AD}$. The intervention also appears to be socially acceptable; one participant stated that 'this has changed my life'. The study highlights the utility and benefits of implementing procedures such as EL, chaining, and prompting and fading with older adults. Although these procedures have a stronger evidence base in clinical contexts with neurodevelopmental disabilities (Cooper et al. 2007); behavioural gerontologists are continuing to develop this area of research with people with dementia (see Trahan et al. 2011; Trahan et al. 2014). The benefits of the CR can be maximised by encouraging participants to engage in regular practice of intervention strategies, and by intervening earlier in the disease course. Future studies should conduct long-term follow-ups and use objective measurement to determine whether $C R$ enhances or maintains performance in ADLs as the disease progresses; and should further investigate possible explanations for post-intervention increases in carer's perceived burden. Research is also required to explore the utility of PT as a strategy for CR.

\section{Acknowledgements}

The authors thank Prof Linda Clare for her advice throughout the study.

\section{Financial Support}

While conducting this research, the first author was employed by the Alzheimer Society of Ireland and The NEIL Programme in Trinity College Dublin, a position funded by the Department of Arts, Heritage, and the Gaeltacht.

\section{Ethical Standards}

The authors assert that all procedures contributing to this work comply with the ethical standards of the relevant national and institutional committee on human experimentation with the Helsinki Declaration of 1975 , as revised in 2008. The study protocol was approved by the institutional review board of the participating institution. Written informed consent was obtained from all participants.

\section{Conflicts of Interest}

The authors declare that there are no conflicts of interest.

\section{References}

Baddeley A, Wilson BA (1994). When implicit learning fails: amnesia and the problem of error elimination. Neuropsychologia 32, 53-68.

Bahar-Fuchs A, Clare L, Woods B (2013). Cognitive training and cognitive rehabilitation for persons with mild to moderate dementia of the Alzheimer's or vascular type: a review. Alzheimer's Research \& Therapy 5, 35.

Barnes-Holmes D, Barnes-Holmes Y, Stewart I, Boles S (2010). A sketch of the implicit relational assessment procedure (IRAP) and the relational elaboration and coherence (REC) model. The Psychological Record 60, 527-542.

Bonomi AE, Patrick DL, Bushnell DM, Martin M (2000). Validation of the United States' version of the World Health Organization Quality of Life (WHOQOL) instrument. Journal of Clinical Epidemiology 53, 1-12.

Clare L (2008). Neuropsychological Rehabilitation: A Modular Handbook. Psychology Press: New York.

Clare L (2010). Cognitive rehabilitation and people with dementia. In J. H. Stone \& M. Blouin (Eds.). International Encyclopedia of Rehabilitation. Buffalo: Center for International Rehabilitation Research Information and Exchange. Retrieved on 14th March 2012 from http://cirrie.buffalo.edu/encyclopedia/en/article/129/.

Clare L, Bayer A, Burns A, Corbett A, Jones R, Knapp M, Kopelman M, Kudlicka A, Leroi I, Oyebode J, Pool J, Woods B, Whitaker R (2013). Goal-oriented cognitive rehabilitation in early-stage dementia: study protocol for a multi-centre single-blind randomised controlled trial (GREAT). Trials 14, 152. 
Clare L, Jones RS (2008). Errorless learning in the rehabilitation of memory impairment: a critical review. Neuropsychology Review 18, 1-23.

Clare L, Linden DE, Woods RT, Whitaker R, Evans SJ, Parkinson CH, van Paasschen J, Nelis SM, Hoare Z, Yuen KS, Rugg MD (2010). Goal-oriented cognitive rehabilitation for people with early-stage Alzheimer disease: a single-blind randomized controlled trial of clinical efficacy. The American Journal of Geriatric Psychiatry 18, 928-939.

Clare L, Wilson BA (2004). Memory rehabilitation techniques for people with early-stage dementia. Zeitschrift für Gerontopsychologie \& Psychiatrie 17, 109-117.

Clare L, Wilson BA, Carter G, Breen K, Gosses A, Hodges JR (2000). Intervening with everyday memory problems in dementia of Alzheimer type: an errorless learning approach. Journal of Clinical and Experimental Neuropsychology 22, 132-146.

Clare L, Wilson BA, Carter G, Hodges JR (2003). Cognitive rehabilitation as a component of early intervention in Alzheimer's disease: a single case study. Aging \& Mental Health 7, 15-21.

Clare L, Wilson BA, Carter G, Roth I, Hodges JR (2002). Assessing awareness in early-stage Alzheimer's disease: development and piloting of the Memory Awareness Rating Scale. Neuropsychological Rehabilitation 12, 341-362.

Clare L, Wilson BA, Carter G, Roth I, Hodges JR (2004). Awareness in early-stage Alzheimer's disease: relationship to outcome of cognitive rehabilitation. Journal of Clinical and Experimental Neuropsychology 26, 215-226.

Clare L, van Paasschen J, Evans SJ, Parkinson C, Woods RT, Linden DE (2009). Goal-oriented cognitive rehabilitation for an individual with mild cognitive impairment: behavioural and neuroimaging outcomes. Neurocase 15, 318-331.

Cooper JO, Heron TE, Heward WL (2007). Applied Behavior Analysis, 2nd edn. Pearson: Upper Saddle River, NJ.

Dong Y, Thompson CL, Huey S, Tan J, Swie Lim LB, Pang W, Li-Hsian Chen C (2013). Test-retest reliability, convergent validity and practice effects of the RBANS in a memory clinic setting: a pilot study. Open Journal of Medical Psychology 2, 4B.

Dunn J, Clare L (2007). Learning face-name associations in early-stage dementia: comparing the effects of errorless learning and effortful processing. Neuropsychological Rehabilitation 17, 735-754.

Fernandez-Ballesteros R, Zamarron MD, Tarraga L (2005). Learning potential: a new method for assessing cognitive impairment. International Psychogeriatrics 17, 119-128.

Folstein MF, Folstein SE, McHugh PR (1975). "Mini-mental state". A practical method for grading the cognitive state of patients for the clinician. Journal of Psychiatric Research 12, 189-198.

Gast DL (2010). Single Subject Research Methodology in Behavioral Sciences. Taylor and Francis: Abingdon, Oxfordshire.

Goldberg D (1992). General Health Questionnaire (GHQ-12). Nfer-Nelson: Windsor.

Grady CL, McIntosh AR, Beig S, Keightley ML, Burian H, Black SE (2003). Evidence from functional neuroimaging of a compensatory prefrontal network in Alzheimer's disease. The Journal of Neuroscience 23, 986-993.
Horner RH, Carr EG, Halle J, McGee G, Odom S, Wolery M (2005). The use of single-subject research to identify evidence-based practice in special education. Exceptional Children 71, 165-179.

Johnson-Talbert C, Cooper JO (1992). Precision teaching and Alzheimer's. Journal of Precision Teaching 10, 53-72.

Kratochwill TR, Hitchcock J, Horner RH, Levin JR, Odom SL, Rindskopf DM, Shadish WR (2010). Single-case designs. Technical documentation. What Works Clearinghouse: http://ies.ed.gov/ncee/wwc/pdf/wwc_scd.pdf.

Kratochwill TR, Hitchcock J, Horner RH, Levin JR, Odom SL, Rindskopf DM, Shadish WR (2013). Single-case intervention research design standards. Remedial and Special Education 34, 26-38.

Kubina RM, Ward MC, Mozzoni MP (2000). Helping one person at a time: precision teaching and traumatic brain injury rehabilitation. Behavioral Interventions 15, 189-203.

Kurz A, Thone-Otto A, Cramer B, Egert S, Frolich L, Gertz HJ, Kehl V, Wagenpfeil S, Werheid K (2012). CORDIAL: cognitive rehabilitation and cognitive-behavioral treatment for early dementia in Alzheimer disease: a multicenter, randomized, controlled trial. Alzheimer Disease and Associated Disorders 26, 246-253.

Lane JD, Gast DL (2014). Visual analysis in single case experimental design studies: brief review and guidelines. Neuropsychological Rehabilitation 24, 445-463.

Lawton MP, Brody EM (1969). Assessment of older people: self-maintaining and instrumental activities of daily living. Gerontologist 9, 179-186.

Lindsley O (1991). Precision teaching's unique legacy from B. F. Skinner. Journal of Behavioral Education 1, 253-266.

Logsdon RG, Gibbons LE, McCurry SM, Teri L (1999). Quality of life in Alzheimer's disease: patient and caregiver reports. Journal of Mental Health and Aging 5, 21-32.

Manolov R, Losada JL, Chacón-Moscoso S, SanduveteChaves S (2016). Analyzing two-phase single-case data with non-overlap and mean difference indices: illustration, software tools, and alternatives. Frontiers in Psychology 7,32 .

Martin M, Clare L, Altgassen AM, Cameron MH, Zehnder F (2011). Cognition-based interventions for healthy older people and people with mild cognitive impairment. Cochrane Database of Systematic Reviews, CD006220.

Nasreddine ZS, Phillips NA, Bedirian V, Charbonneau S, Whitehead V, Collin I, Cummings JL, Chertkow H (2005). The Montreal Cognitive Assessment, MoCA: a brief screening tool for mild cognitive impairment. Journal of the American Geriatrics Society 53, 695-699.

Oren S, Willerton C, Small J (2014). Effects of spaced retrieval training on semantic memory in Alzheimer's disease: a systematic review. Journal of Speech Language and Hearing Research 57, 247-270.

O'Sullivan M, Coen R, O'Hora D, Shiel A (2015). Cognitive rehabilitation for mild cognitive impairment: developing and piloting an intervention. Aging Neuropsychology and Cognition: A Journal on Normal and Dysfunctional Development 5, 280-300.

Randolph C, Tierney MC, Mohr E, Chase TN (1998). The Repeatable Battery for the Assessment of Neuropsychological 
Status (RBANS): preliminary clinical validity. Journal of Clinical and Experimental Neuropsychology 20, 310-319.

Ready RE, Ott BR (2003). Quality of life measures for dementia. Health and Quality of Life Outcomes 1, 11.

Sitzer DI, Twamley EW, Jeste DV (2006). Cognitive training in Alzheimer's disease: a meta-analysis of the literature. Acta Psychiatrica Scandinavica 114, 75-90.

Shadish WR, Rindskopf DM (2007). Methods for evidencebased practice: quantitative synthesis of single-subject designs. New Directions for Evaluation 113, 95-109, https:/ / doi.org/10.1002/ev.217.

Shadish WR, Sullivan KJ (2011). Characteristics of single-case designs used to assess intervention effects in 2008. Behavior Research Methods 43, 971-980.

Shadish WR (2014). Statistical analysis of single-case designs: the shape of things to come. Current Direction in Psychological Science 23, 139-146.

Skinner BF (1953). Science and Human Behavior. Macmillan: Oxford, England.

Suhr J (1999). Progressive muscle relaxation in the management of behavioural disturbance in Alzheimer's disease. Neuropsychological Rehabilitation 9, 31-44.

Tate RL, Perdices M, McDonald S, Togher L, Rosenkoetter U (2014). The design, conduct and report of single-case research: resources to improve the quality of the neurorehabilitation literature. Neuropsychological Rehabilitation 24, 315-331.
Tate R, McDonald S, Perdices M, Togher L, Schultz R, Savage $S$ (2008). Rating the methodological quality of single-subject designs and n-of-1 trials: introducing the Single-Case Experimental Design (SCED) Scale.

Neuropsychological Rehabilitation 18, 385-401.

Tate RL, Perdices M, Rosenkoetter U, Wakim D, Godbee K, Togher L, McDonald S (2013). Revision of a method quality rating scale for single-case experimental designs and n-of-1 trials: the 15-item Risk of Bias in N-of-1 trials (RoBiNT) Scale. Neuropsychological Rehabilitation 23, 619-638, doi: http:/ /dx. doi.org/ 10.1080/09602011.2013.824383.

Terrace HS (1963). Discrimination learning with and without "errors". Journal of the Experimental Analysis of Behavior 6, $1-27$.

Trahan MA, Kahng S, Fisher A, Hausman NL (2011). Behavior analytic research on dementia in older adults. Journal of Applied Behavior Analysis 44, 687-691.

Trahan MA, Kuo J, Carlson M, Gitlin LN (2014). A systematic review of strategies to foster activity engagement in persons with dementia. Health Education and Behaviour 41 (Suppl.): 70S-83S.

Zarit S, Orr NK, Zarit JM (1985). The Hidden Victims of Alzheimer's Disease: Families Under Stress. New York University Press: New York.

Zigmond AS, Snaith RP (1983). The hospital anxiety and depression scale. Acta Psychiatrica Scandinavica 67, 361-370. 